\title{
The predictive value of fruit juice in the esophagus-pleural fistula
}

\author{
Lucio Brugioni, ${ }^{1}$ Francesca De Niederhausern, ${ }^{1}$ Elisa Romagnoli, ${ }^{1}$ Beatrice Aramini, ${ }^{2}$ Massimo Girardis,${ }^{3}$ \\ Angela Bertani, ${ }^{4}$ Francesco Fontana, ${ }^{5}$ Manuel Gramolini ${ }^{6}$ \\ ${ }^{1}$ Internal Medicine, Critical Area Unit, Policlinico Modena; ${ }^{2}$ Division of Thoracic Surgery, Policlinico Modena; ${ }^{3}$ Intensive \\ Care Unit, Policlinico Modena; ${ }^{4}$ Endoscopy Service, Policlinico Modena; ${ }^{5}$ Nephrology, Policlinico Modena; ${ }^{6}$ Emergency \\ Medicine, University of Modena and Reggio Emilia, Italy
}

\begin{abstract}
Esophageal-pleural fistula is a rare and challenging condition to diagnose and requires strong clinical suspicion in order to be recognized promptly. Chest computed tomography (CT) with contrast medium for oral contrast medium (OS) is the gold standard for diagnosis. The definitive therapy is purely surgical, except for a few selected cases that benefit from endoscopic therapy. Our case involves a 45-year-old woman who came to the Emergency Department with dyspnea and thoracalgia. Chest $\mathrm{X}$-ray and high-resolution CT showed empyema and pleural effusion to the left hemithorax. The lack of improvement despite the therapy and a subsequent clinical finding gave rise to the suspicion of esophageal-pleural fistula, confirmed with CT with contrast medium for OS. In this case, we opted for endoscopy correction of the esophageal defects. The diagnostic delay and the pre-existing comorbidities (previous kidney transplant for chronic kidney disease from lupus nephritis, high blood pressure, familiarity with Ischemic cardiomyopathy) could justify the inauspicious course of our case.
\end{abstract}

\section{Introduction}

Esophageal-pleural fistula is a rare and challenging condition to diagnose. The etiology is varied, most esophageal-pleural fistulas are iatrogenic (mainly from surgical or endoscopic complications); spontaneous

Correspondence: Lucio Brugioni, Internal Medicine, Critical Area Unit, Policlinico Modena, via del Pozzo 71, 41121 Modena, Italy.

Tel.: +39.059.422549.

E-mail: brugioni.lucio@aou.mo.it

Key words: Esophageal-pleural fistula; empyema; chest computed tomography; endoscopic therapy; mini-thoracotomy.

Contributions: all authors contributed equally to the writing of the article.

Conflict of interests: the authors declare no potential conflict of interests.

Ethics approval: not required.

Informed consent: the patient gave informed consent.

Received for publication: 7 April 2020.

Revision received: 1 May 2020

Accepted for publication: 4 May 2020.

This work is licensed under a Creative Commons Attribution NonCommercial 4.0 License (CC BY-NC 4.0).

${ }^{\circ}$ Copyright: the Author(s), 2020

Licensee PAGEPress, Italy

Italian Journal of Medicine 2020; 14:176-178

doi:10.4081/itjm.2020.1285 rupture (or Boerhaave syndrome) is the most frequent non iatrogenic cause, more rarely they are due to ingestion of extraneous bodies, mainly in the pediatric patient, barotrauma and penetrating trauma, neoplasms, esophagitis, ulcers, and infections. ${ }^{1-4}$ The clinical analysis is not typical and may be characterized by chest pain, loss of appetite and vomiting, dyspnea, signs and symptoms of pneumomediastinum, and/or pleural effusion. The gold standard for diagnosis is chest computed tomography (CT) with contrast medium for oral contrast medium (OS) or diagnostic-operative endoscopy in unstable patients. X-ray and chest ultrasound can confirm a clinical suspicion, with pneumomediastinum relief, subcutaneous emphysema, pleural effusion, and hydropneumothorax (PNX). Laboratory tests can show leukocytosis; the analysis of pleural fluid can be useful, with the detection of traces of food, high level of salivary amylase, and the presence of squamous epithelial cells. Complications of unrecognized or early treated esophageal-pleural fistula include empyema, mediastinitis, aspiration pneumonia, and cardiac tamponade. ${ }^{1,5,6}$ Medical therapy should be reserved for patients with limited perforation and in the cervical or mediastinal area, in the absence of obstructions and sepsis. It provides oral fasting and nutrition through a nasogastric tube or total parenteral nutrition, proton-pump inhibitors, and broad-spectrum and antifungal antibiotics, eventual drainage of the collected fluid. Surgical therapy with the repair of the defect by thoracoplasty is the therapy of choice, endoscopic treatment with stent positioning is reserved for patients with important comorbidities who could not tolerate surgery. ${ }^{7,8}$ 


\section{Case Report}

A 45-year-old woman came to ED with chest pain, dyspnea, and dyspepsia. In anamnesis, she had a kidney transplant for chronic kidney disease from lupus nephritis in antirejection and steroid therapy, high blood pressure in drug therapy, familiarity with Ischemic cardiomyopathy. The vital signs showed Glasgow Coma Scale 15 , temperature $36.0^{\circ} \mathrm{C}$, tachodyspnea with $90 \%$ oxygen saturation, arterial pressure $100 / 60 \mathrm{mmHg}$ with $85 \mathrm{bpm}$ in $\beta$-blocker therapy. There was objectively shallow breathing with vesicular murmur reduced to the left field, rhythmic heart tones, treatable and indolent abdomen. The electrocardiogram did not show anything relevant. Laboratory tests showed mild anemia with moderate to severe thrombocytopenia, acute renal failure, elevated cardiac enzymes, indices of inflammation. The chest X-ray (Figure 1) showed a large left hydroPNX layer with a mediastinal shift. The thoracic tube was positioned with draining of air and $1500 \mathrm{cc}$ of turbid liquid. Due to rapid hemodynamic deterioration, the patient was intubated, supported with volemic and amine filling, and hospitalized in intensive care. During the 29 days of hospitalization in intensive care, further radiological and laboratory tests were carried out. All viral serologies and microbial cultures (blood, urinary) were negative, except for the growth of Streptococcus salivarius in the pleural fluid taken from thoracic drainage. From the thoracic drainage, remained a continuous and constant leakage of serum-turbid liquid. The chestabdomen CT with intravenous contrast showed the evolution of the picture with the appearance of hydroPNX and empyematous sacchae to the left hemithorax, for which we proceeded to the surgical toilet with mini-thoracotomy. Despite hemodynamic support therapy, broad-spectrum antibiotic therapy, and surgical correction of the empyema, the patient remained critical, and the markers of sepsis persisted in the laboratory data. During a period of weaning from mechanical ventilation, the patient assumed for OS a fruit juice, and the medical staff noticed the leakage of the same in the thoracic drainage in place. This event aroused a new clinical suspicion, and chest CT was performed with contrast medium OS, which confirmed the diagnostic suspicion of an esophagealpleural fistula with spreading of the contrast medium before the thoracic aorta from continuity solution of the lateral esophageal wall in the left pleural cavity (Figure 2). Afterward, operative esophagogastroduodenoscopy was performed with the positioning of a metal stent and effective closure of the breach. However, the clinical conditions did not show improvement, and the progression of multiple organ failures (MOFS) was expected with the development of different antibiotic resistances in the various crops. The patient was transferred to a third level center specialized in the treatment of MOFS: another chest CT with contrast medium OS showed persistent esophageal-pleural fistula that was subject to surgical correction before metal esophageal stent removal. Residual esophageal stenosis was treated with endoscopic balloon dilatation and stent insertion: this procedure was complicated by hematemesis (major bleeding) and related type II myocardial infarction. The patient was discharged after 5 months with percutaneous endoscopic gastrostomy in place for nutritional support.

\section{Discussion}

Our patient presented at the Emergency Department with nonspecific symptoms, such as

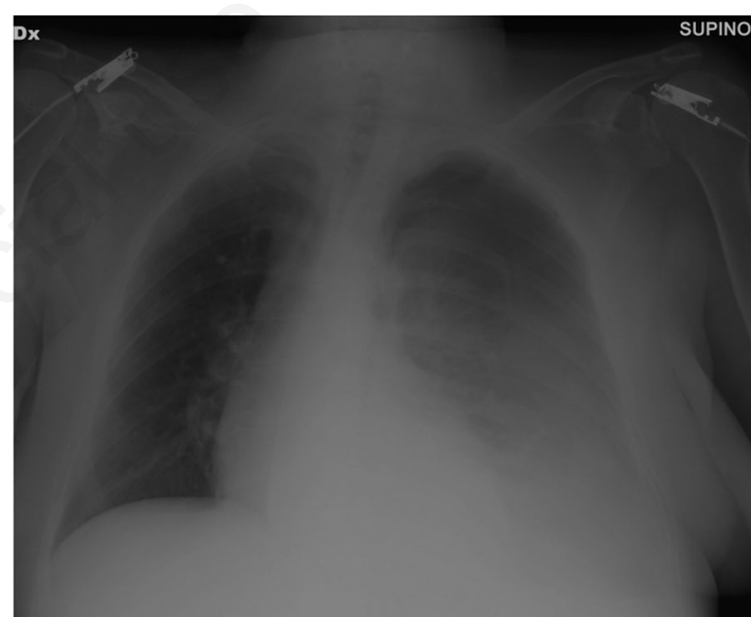

Figure 1. Chest x-ray.

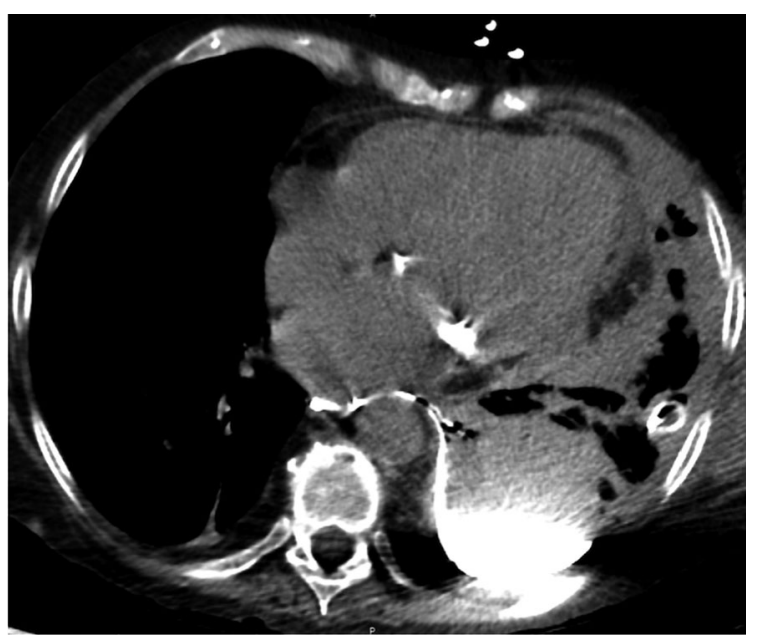

Figure 2. Chest computed tomography. 
thoracalgia and dyspnea, and the radiological picture showed a left pleural effusion but did not raise the suspicion of esophageal-pleural fistula. Critical clinical conditions dictated an intensive resuscitation treatment that initially placed the search for possible differential diagnoses in the background. Only after the detection of traces of food in the thoracic drainage the presence of esophageal-pleural fistula was suspected, and subsequent diagnostic and therapeutic investigations were started. The delay in arriving at the correct diagnosis and treatment could also explain the long and complex evolution of the case.

Findings of food traces in the thoracic drainage already in place, as input for diagnostic suspicion, is not uncommon for this type of pathology and, in literature, other cases have been reported with the relief of milk and/or rice in the pleural fluid. ${ }^{3,6,9}$

Hence the positive predictive value of fruit juice in the diagnosis of the esophagus-pleural fistula is near $100 \%$. On the other hand, a negative predictive value cannot be defined, since the possibility of falsenegative test is almost unlimited.

Even the finding of an oropharynx pathogen, such as $S$. salivarius, in the pleural fluid of thoracic drainage, should have raised the clinical suspicion of a continuous connection between the gastroenteric tract and the pleural cavity. ${ }^{3}$

In the absence of clinical suspicion, the use of standard diagnostics, such as ultrasound, radiology, and CT with intravenous contrast medium, did not help the doctor in the definitive diagnosis because there were no pathognomonic findings of esophagealpleural fistula.

As per guidelines, chest $\mathrm{CT}$ with contrast medium for OS allowed, instead, a correct diagnosis. In our case, the therapeutic process was conditioned by the patient's clinical instability, for which a surgical correction was not proposed. On the contrary, an endoscopically self-expanding metal stent was positioned considering the absence of the most frequent contraindications to this intervention (multiple breaches, lesion near the gastroesophageal junction, breach $>6 \mathrm{~cm}$ long) and the absence of neoplastic tissue and/or obstructions of the bowel. ${ }^{7,8}$

What makes our clinical case interesting is the location of the esophageal-pleural fistula. Anatomically, the esophagus is in direct contact with pleura for a large area in the right hemithorax, while aorta is interposed between the esophagus and pleura on the left. The presence of a left esophageal-pleural fistula, therefore, represents a rarity in a pathology that is in itself rare. ${ }^{1,2}$

The esophageal-pleural fistula in this patient could recognize a multi-pathogenetic mechanism associated with her autoimmune disease. Patients with systematic lupus erythematosus often have an esophageal motility disorder, an inflammatory reaction of the esophageal muscle tunic due to the deposition of immune complexes and an ischemic alteration of the Auerbach plexus related to vasculitis. The risk of infectious esophagitis associated with immunosuppressive therapy is also increased. ${ }^{9}$

\section{Conclusions}

Esophageal-pleural fistula is a rare disease that requires high clinical suspicion to diagnose and establish the proper treatment promptly. Chest CT with contrast medium for OS represents the gold standard for diagnosis. Surgical correction of the breach is the treatment of choice, reserving endoscopy for a few selected cases or more unstable patients. If recognized and treated early, the esophageal-pleural fistula has a mortality of $<10 \%$, while if the diagnosis is delayed beyond $48 \mathrm{~h}$ the prognosis becomes severe. ${ }^{2,3}$ For the clinician in general, but also for the doctor of the emergency department, it is therefore useful to know the fundamentals of this rare pathology to be able to insert the esophageal-pleural fistula into the differential diagnosis scheme, and based on clinical suspicion, to prepare the most correct and timely diagnostic-therapeutic management.

\section{References}

1. Triadafilopoulos G. Boerhaave syndrome: Effort rupture of the esophagus. In: UpToDate, Post, TW (Ed), UpToDate, Waltham, MA, 2020.

2. Raymond, DP. Overview of esophageal perforation due to blunt or penetrating trauma. In: UpToDate, Post, TW (Ed), UpToDate, Waltham, MA, 2020.

3. Dash M, Mohanty T, Patnaik J, et al. An unusual case of spontaneous esophagopleural fistula. Lung India 2017;34:287-9.

4. Cui Y, Ren Y, Zhang Y, et al. Pediatric esophagopleural fistula Two case reports and a literature review, Medicine (Baltimore) 2017;96:e6695.

5. Sameer V, Mahesh P, Niranjan K, et al. Spontaneous oesophago-pleural fistula. Lung India 2011;28:300-2.

6. Mishra VK, Singh RK, Chaudhuri S, Singh AK. Incessant pleural drainage - Can it be a masquerading esophageal-pleural fistula?. Indian J Respir Care 2019;8:60-2.

7. Raymond DP. Surgical management of esophageal perforation. In: UpToDate, Post, TW (Ed), UpToDate, Waltham, MA, 2020.

8. Kang GH, Yoon BY, Kim BH, et al. A case of spontaneous esophagopleural fistula successfully treated by endoscopic stent insertion. Clin Endosc 2013;46:91-4.

9. Massarotti EM. Gastrointestinal manifestations of systemic lupus erythematosus. In: UpToDate, Post, TW (Ed), UpToDate, Waltham, MA, 2020. 